\title{
Laser Action Studies of $\pi$-Conjugated Polymer Microcavities
}

\author{
A. Tulek ${ }^{1}$ and Z.V. Vardeny ${ }^{2}$ \\ ${ }^{I}$ Department of Physics, Bilkent University, 06800 Bilkent, Ankara, Turkey \\ ${ }^{2}$ Department of Physics, University of Utah, Salt Lake City, Utah 84112, USA \\ Tel: (90312) 2903506, Fax :( 90312)2664365, e-mail: tulek@unam.bilkent.edu.tr
}

\begin{abstract}
Unidirectional laser emission was observed from $\pi$-conjugated polymer microcavities with spiral geometry, and from microdisks containing line defects. For both type of cavities directionality contrast of the laser emission was found to be $\sim 10$, with far-field lateral divergence angle of $\sim 15^{\circ}$. No significant increase in the laser threshold intensity was observed compared with simple microdisk cavities. In addition we also studied the emission spectra of microring polymer lasers fabricated on nylon microfibers upon application of uniaxial stress with strain up to $\sim 12 \%$. Substantial change in the laser emission spectrum was observed when stress was applied; showing enhanced optical sensitivity to stress larger than the changes induced in the emission spectrum caused by the microfiber diameter change. We explain the enhanced sensitivity to stress as due to the induced change in the polymer refractive index dispersion, in particular at the laser emission wavelength $(\sim 635 \mathrm{~nm})$.
\end{abstract}

Keywords: laser action, microcavity, whispering gallery modes, directional emission, $\pi$-conjugated polymers, uniaxial stress.

\section{INTRODUCTION}

Light in a microcavity is confined by total internal reflection at the boundary due to discontinuity of the refractive index, $n$, at the interface between the active medium and its surrounding. Such a morphologydependent light confinement provides the essential positive resonant feedback needed for laser action. Many studies have been accomplished to characterize such microlasers due to their ease of fabrication and high quality factor. In addition to inorganic gain media, organic microlasers have been also extensively studied during the last decade, and laser action was demonstrated using various organic materials such as laser dyes [1] and $\pi$-conjugated polymers [2].

In our research group we have studied organic microlasers with two goals in mind. One goal has been to obtaining unidirectional laser emission by breaking the circular symmetry of microdisks. The second goal has been to measure the change in the effective refractive index of the gain medium when subject to a uniaxial stress, by observing the corresponding shift in the laser emission spectrum from microrings.

\section{UNIDIRECTIONAL LASER EMISSION FROM $\pi$-CONJUGATED POLYMER MICROCAVITIES}

For the gain medium in our studies we have used the polymer poly(dioctyloxy) phenyl vinyline [DOO-PPV], a red-emitter $\pi$-conjugated polymer with high photoluminescence emission quantum efficiency. The polymer was dissolved in toluene and spin-cast onto a glass substrate forming a uniform film with thickness of $\sim 2 \mu \mathrm{m}$. Microcavities with unorthodox geometries such as spiral and disk with specific line defect were subsequently patterned onto the polymer film using conventional lithographic techniques.

The spiral geometry was defined according to the relation: $r(\phi)=r_{0}(1+\varepsilon \phi / 2 \pi)$, where $r_{0}$ is the smallest radius and $\varepsilon$ is the deformation factor. $r_{0}$ in the fabricated microcavities ranged from 50 to $150 \mu \mathrm{m}$, and $\varepsilon$ varied from 0.15 to 0.30 . Line defects in the polymer microdisks were fabricated with a fixed width of $2 \mu \mathrm{m}$ and length of 10 and $20 \mu \mathrm{m}$. The distance from the defect edge to the microdisk boundary was $\sim 10 \mu \mathrm{m}$ in all cases. The orientation of the line defect with respect to the circular geometry was characterized by an angle $\theta$ (Fig. 2(b) inset) and set to $\theta=45^{\circ}, 60^{\circ}$ and $75^{\circ}$, respectively.

The microcavities were optically excited using the second harmonic of a pulsed Nd:yttrium-aluminum-garnet laser amplifier system operating at $532 \mathrm{~nm}$, with pulse duration of $100 \mathrm{ps}$ at $870 \mathrm{~Hz}$ repetition rate. The laser emission was collected using a glass fiber of $1 \mathrm{~mm}$ diameter placed at $\sim 1 \mathrm{~cm}$ away from the microcavity, and subsequently sent to a triple spectrometer, where a CCD camera recorded the light intensity. The overall spectral resolution of the collected light emission was $\sim 0.1 \mathrm{~nm}$. A manual rotational stage having $2^{\circ}$ accuracy was used to rotate the microstructures for azimuthal intensity distribution measurements, and the recorded intensity was plotted versus the azimuthal angle, $\varphi$.

Spiral microcavity: The emission vs. excitation intensity dependence is shown in Fig. 1(a) inset; the threshold intensity, $I_{\text {th }}$ of the spiral cavity with $r_{0}=79 \mu \mathrm{m}$ and $\varepsilon=0.30$ was $\sim 100 \mathrm{~nJ} / \mathrm{pulse}$; comparable to that of a corresponding microdisk $(\sim 90 \mathrm{~nJ} / \mathrm{pulse})$. This spiral microcavity clearly shows unidirectional emission (Fig. 1(b)). The laser emission has a narrow lateral divergence angle (LDA) of $\sim 12^{\circ}$; and high directionality contrast (DC) of $\sim 10$, which improves at high excitation intensities, and larger cavity deformation factors. 
(a)

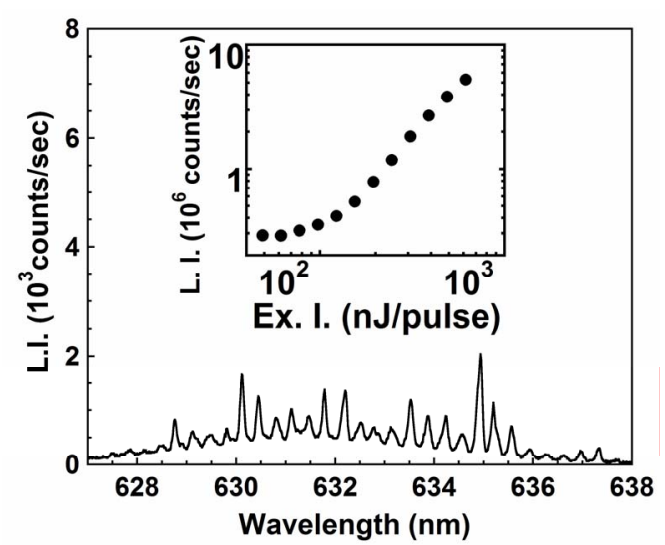

(b)

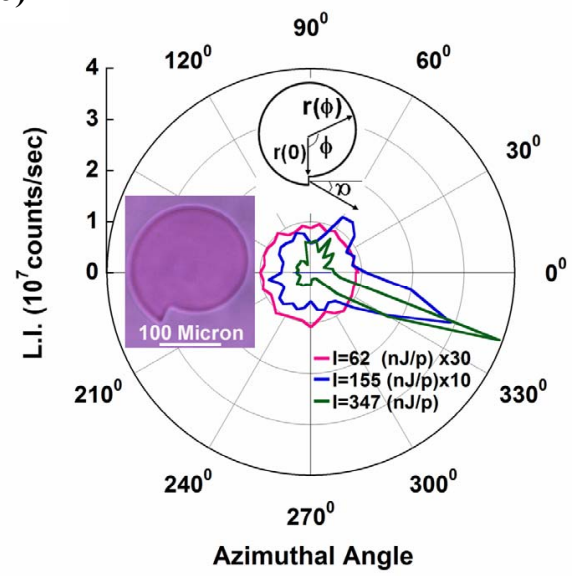

Figure 1. (a) The laser emission spectrum of a DOO-PPV micro-spiral with $\varepsilon=0.30$. The inset is the laser emission vs. excitation intensity, showing $I_{t h} \sim 100 \mathrm{~nJ} /$ pulse. (b) The azimuthal emission intensity distribution at $I<I_{\text {th }}$ (red line) and $I>I_{\text {th }}$ (green and blue lines). The left inset is the optical image of the cavity with $r_{0}=79 \mu \mathrm{m}$. The top inset shows the geometrical definitions of the spiral micro-cavity (see text).

Microdisk with line defect: Fig. 2 shows that $I_{t h}$ is $\sim 100 \mathrm{~nJ} /$ pulse, independent of the fabricated line defect parameters. In addition the emission spectrum preserves its ordered structure produced by the disk shape (Fig. 2(a)), indicating that the whispering gallery modes (WGM) in such cavities are not substantially disturbed by the fabricated linear defect. Unidirectional emission was observed from such microstructure with DC of $\sim 8$ and LDA of $\sim 15^{\circ}$ (Fig. 2(b)). No considerable difference in terms of directionality was observed with respect to the size, or orientation of the line defect. We note that the laser mode linewidth for this type of cavity is the same as in plain microdisks showing that the microlaser quality factor is determined by impurities, defects, self absorption and inhomogeneity in the polymer film, rather than by the cavity quality factor.

(a)

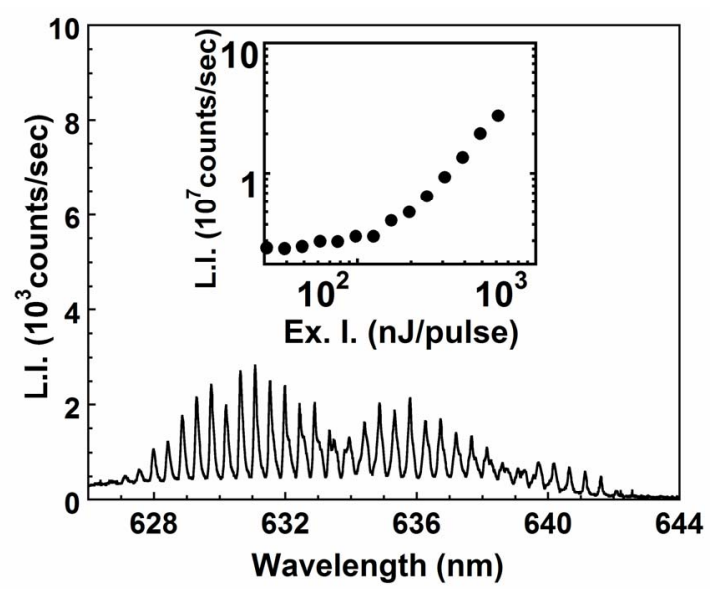

(b)

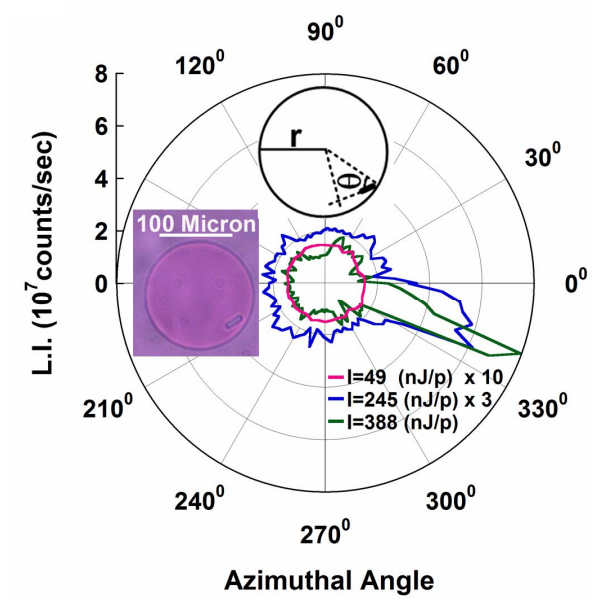

Figure 2. Same as in Fig. 1 but for a microdisk with diameter D = $155 \mu \mathrm{m}$ having a line defect of $2 \mu \mathrm{m} \times 20 \mu \mathrm{m}$, with $\theta=60^{\circ}$ (see top inset).

\section{POL YMER MICRORING LASERS SUBJECT TO UNIAXIAL STRESS}

Since organic gain media are mechanically flexible, they easily respond to moderate external forces such as stress, while maintaining laser action. This flexibility may be used for monitoring the organic microcavity deformation and the associate change in the effective refractive index, $n_{\text {eff, }}$ of the gain medium upon stretching, by studying the laser emission spectrum. This is possible since the longitudinal laser mode separation directly depends on $n_{\text {eff }}$ and cavity size [3]. Here we report a large decrease in $n_{\text {eff }}$ with stress, which leads to enhanced sensitivity of $\pi$-conjugated polymer gain media for monitoring stress. The following relation between $n_{\text {eff }}$ dispersion and the laser mode separation explains the laser sensitivity to the applied stress [3]: 


$$
n_{\text {eff }}=n-\lambda \frac{d n}{d \lambda}
$$

where $\lambda$ is the lasing wavelength and $\lambda d n / d \lambda$ is the first order dispersion term. We demonstrated that the dispersion term in Eq. (1) changes much more than $n$ upon stretching the polymer film and this provides the improved sensitivity of the medium for detecting stress.

The polymer microring lasers were fabricated by drop casting the polymer solution onto nylon microfibers with diameter in the range of $35-90 \mu \mathrm{m}$. The coated fiber was subsequently mounted onto a specially engineered stretching unit which could apply a predetermined force on the fiber, such that the fiber length, $L$ was increased by increments of $0.5 \mathrm{~mm}$. The new fiber length, $L_{f}$ and diameter $D_{f}$ were monitored in situ by an optical microscope. The microring cavities were optically excited and the emission was collected with the same experimental setup mentioned above. In addition all measurements were performed under vacuum conditions of $\sim 10^{-3}$ Torr to avoid photo-oxidation of the DOO-PPV polymer.

The power Fourier transform (PFT) is a useful tool in monitoring the mode spacing in the emission spectrum, since it contains equally spaced, discrete FT components with separation, $\Delta d$ given by the relation [3]:

$$
\Delta d=n_{\text {eff }} D / 2 \text {, }
$$

where $d$ is the FT 'length' parameter. We therefore monitored the change in $n_{\text {eff }} D$ parameter of the microring cavities upon stretching by studying the laser emission PFT using Eq. (2).

Fig. 3(a) shows the change in the emission spectrum of a $37 \mu \mathrm{m}$ diameter microring laser induced upon uniaxial stretch up to $\sim 12 \%$ strain; the corresponding PFT of the emission spectra are shown in Fig. 3(b). Although there is little change in the laser threshold intensity upon stretching, there is a large change in the laser emission spectrum. To better present the shift of the discrete FT components in the PFT upon stretching, we emphasize the induced change of the $4^{\text {th }}$ harmonic by arrows. It is clearly seen that the discrete FT components shift and thus $\Delta d$ decreases, in agreement with Eq. (2). However the change in $\Delta d$ is much larger than that expected from the stress induced change in $D$, when using the induced strain and Poisson ratio to calculate $\Delta D$. When Eq. (2) is applied to stress-free microring lasers with various diameters we found that $n_{\text {eff }}$ is $\sim 1.74$, and independent on $D$. This shows that the stressed induced change in $n_{\text {eff }}$ does not come from a change in $D$, but is rather due to an induced change in the polymer optical constants.

(a

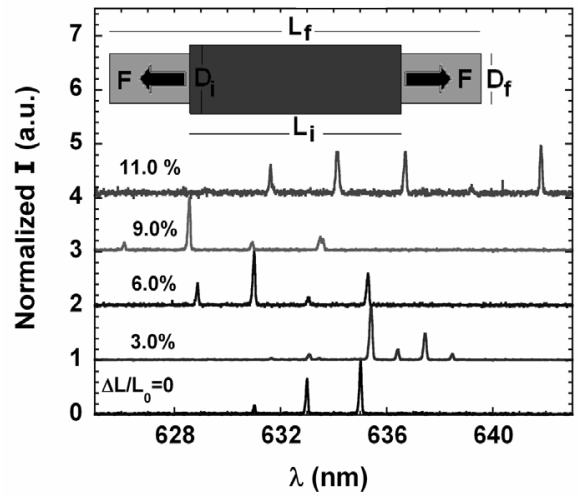

(b)

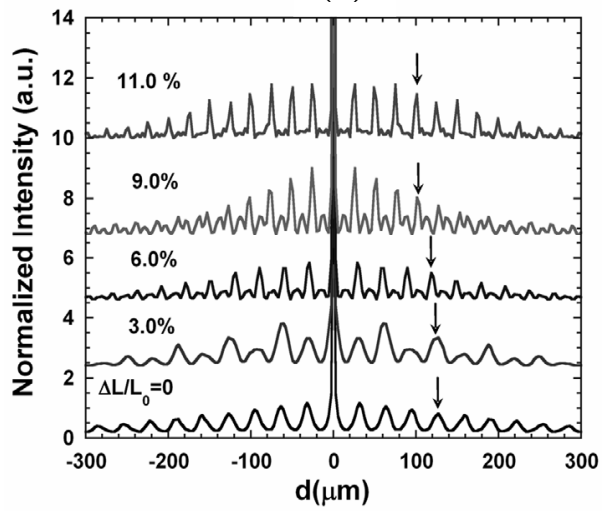

Figure 3. Laser emission spectra (a) and corresponding PFT (b) of a polymer microring laser subject to uniaxial stress, with strain up to $12 \%$. The arrows in (b) point to the fourth discrete FT harmonics. The inset in (a) shows the stretching direction, the initial $\left(L_{i}\right)$ and final $\left(L_{f}\right)$ lengths, and diameters, $D_{i}$ and $D_{f}$, respectively.

The change in the laser parameter $n_{e f f} D$ calculated from Fig. 3(b) using Eq. (2), with the corresponding change in the fiber diameter $D$ (measured by an optical microscope) as a function of the applied longitudinal strain, $\Delta L / L_{0}$ is shown in Fig. 4 (a). It is seen that the induced change in $n_{e f f} D$ is much larger than that of $D$. By comparing the two sets of data, we calculated the induced change in the laser $n_{\text {eff }}$ as illustrated in Fig. 4(b). A large decrease in $n_{\text {eff }}$ is realized with stretching that is roughly the same as $\sim 12 \%$ stretch induced change in $D$. The changes in $n_{\text {eff }}$ and $D$ then add up for a total of $\sim 25 \%$ decrease in the value of the laser $n_{\text {eff }} D$ parameter with stretching; this explains the superior sensitivity of the polymer lasers to monitor stress.

In order to understand the dramatic change in $n_{\text {eff }}$ with stretching, $d n / d \lambda(\lambda)$ and $n(\lambda)$ spectra were measured using the optical transmission spectrum, $T(\lambda)$ (Fig. 4 (b)) for a stretched $\left(\Delta L / L_{0} \sim 5 \%\right.$ ) polymer film with both polarizations parallel and perpendicular to the stretching direction. For obtaining $n(\lambda)$ spectrum the complex refractive index $\boldsymbol{n}=n+\mathrm{i} k$ was calculated from $T(\lambda)$ using the effective medium approximation method [4]. We realized that $n(\lambda)$ becomes anisotropic upon stretching. For polarization perpendicular to the stretching direction 
very little change in $n(\lambda)$ is observed. However for polarization parallel to the stretching direction we observed that $n(\lambda)$ spectrum broadens and red-shifts upon stretching; consequently the dispersion $d n / d \lambda$ dramatically increases at the laser emission wavelength $\left(\lambda_{0} \sim 635 \mathrm{~nm}\right)$. The induced changes in $n(\lambda)$ upon stretching are probably due to reversible chain reorientation in the stretching direction. For microring lasing, the change in $n(\lambda)$ parallel is more important since the emission is polarized in this direction [5]. In fact when calculating $n_{\text {eff }}$ parallel (at $635 \mathrm{~nm}$ ) using Eq. (1), $n=1.73$, and $\lambda d n / d \lambda=0.07$ we obtained $n_{\text {eff }}=1.66$ for the stretched polymer film at its maximum stretching length. Assuming that $\lambda d n / d \lambda$ increases linearly with the applied strain, and taking into account that the measured film was subjected to a strain of $\sim 5 \%$, then a reduction in $n_{\text {eff }}$ of about $0.17(\sim 10 \%)$ is expected for a $12 \%$ strain. This agrees with the obtained reduction of $\sim 12 \%$ in $n_{\text {eff }}$ for the stretched polymer microcavity when a $12 \%$ strain is applied, explaining the sensitivity of polymer lasers to monitor stress via $n_{e f f}$.
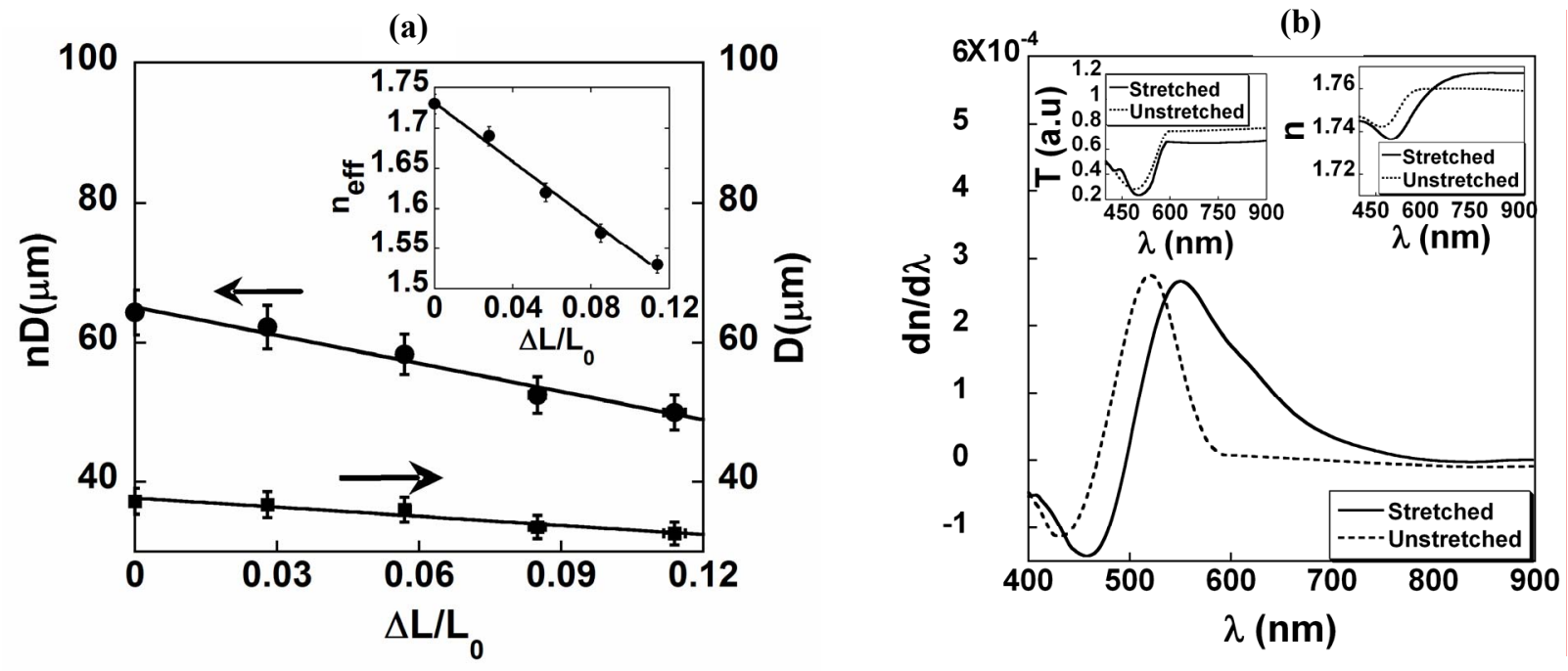

Figure 4. (a) The stress-induced change in the laser parameter $n_{\text {eff }} D$ (circles; left scale) obtained from the analysis of the emission spectra of Fig. 3 (b) using Eq. (2); and the corresponding changes in D (squares; right scale) measured by an optical microscope. Inset is the calculated change in $n_{\text {eff }}$ induced upon stretching as obtained from the data. (b) The refraction index dispersion spectrum dn/d $\lambda$ of an unstretched and stretched polymer film on a nylon substrate. The right inset shows the corresponding change in $n(\lambda)$ spectrum, and left inset shows the measured transmission spectrum upon stretching.

\section{CONCLUSIONS}

In conclusion, we studied two applications of organic microlasers based on DOO-PPV polymer as gain medium. We found that the fabricated microstructures with broken symmetry exhibited very promising unidirectional laser emission. The improved emission directionality did not come at the expense of $I_{t h}$, therefore directional emission in polymer lasers can be obtained without a substantial increase in the excitation intensity. The microdisk cavity containing line defect has an advantage over the spiral microcavity, since it preserves the equidistant mode spacing in the emission spectrum along with similar improved directionality, and is easier to fabricate. A substantial decrease has been observed in the effective refractive index of the gain medium in microring lasers fabricated from $\pi$-conjugated polymer operating under uniaxial stress. The enhanced $n_{\text {eff }}$ sensitivity to the applied stress is attributed to the induced change in the polymer refractive index dispersion in the direction parallel to the stretching direction.

\section{REFERENCES}

[1] M. Kuwata-Gonokami, R. H. Jordan, A. Dodabalapur, et al.: Polymer microdisk and microring lasers, Opt. Lett., vol. 20, pp. 2093-2095, October 1995.

[2] A. Tulek and Z.V.Vardeny: Unidirectional laser emission from pi-conjugated polymer microcavities

[3] with broken symmetry, Appl. Phys. Lett., vol. 90, pp. 161106-3, April 2007.

[4] A. Tulek and Z.V.Vardeny: Studies of polymer microring lasers subject to uniaxial stress, Appl. Phys. Lett., vol. 91, pp. 121102-3, September 2007.

[5] S. Bosch, J. Ferre'-Borrull, N. Leinfellner et al: : Effective dielectric function of mixtures of three or more materials: a numerical procedure for computations, Surf. Sci., vol. 453, pp. 9-17, February 2000.

[6] S. V. Frolov, A. Fujii, D. Chinn, et al.: Cylindrical microlasers and light emitting devices from conducting polymers, Appl. Phys. Lett., vol. 72, pp. 2811-2813, June 1998. 